\title{
TOOLS AND METHODS OF TELEMEDICINE FOR EARLY DETECTION OF DENTAL ANOMALIES
}

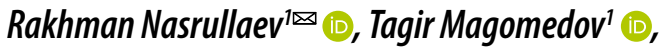 Gleb Mareev ${ }^{2}$ (D), Anna Petrova ${ }^{1}$, Olga Popkova ${ }^{1}$ (D), Irina Firsova ${ }^{1}$ (D), Valery Konnov ${ }^{3}$ (D)}

\author{
${ }^{1}$ Department of Pediatric Dentistry and Orthodontics, Saratov State \\ Medical University, Saratov \\ ${ }^{2}$ Department of Otorhinolaryngology, Saratov State Medical University, \\ Saratov \\ ${ }^{3}$ Department of Orthopedic Dentistry, Saratov State Medical University, \\ Saratov, Russia
}

\section{$\triangle$ nasrullaevrk@yandex.ru}

Received 23 July 2020;

Received in revised form 27 August 2020; Accepted 31 August 2020
ABSTRACT - This work offers a view at the practical application of telemedicine, especially in rural areas. There is a description and the principle of independent development. The item also contains comparative data on the effectiveness of jaw model analysis performed in the conventional way as matched against doing that with new software employed to detect dental anomalies, with no previously acquired skills. The software can be used by any doctor as it features detailed and clear instructions. This program will be useful in cases where orthodontists are not available.

KEY W ORDS - telemedicine, diagnostic software, teledentistry, dentoalveolar issues detection.

\section{NTRODUCTION}

Availability of narrowly specialized medical care is an important feature attributing to its quality [7-11]. While in urban areas a specialist's consultation is available to most of the population, rural residents face certain deficits in this respect $[1,2]$. Not only rural areas have a worse access to medical centers, but also a shortage of specialists and the insufficient technological infrastructure present a challenge there $[3,4]$. The advance in communication technologies in remote areas is still slow compared to what it could be nowadays.

Telemedicine is one of the healthcare arrangements that may serve to improve medical services offered in remote areas that face issues with access to specialized care and appropriate equipment $[5,6]$.
Aim of study:

to investigate potential use of telemedicine technologies and to develop software for early detection of dental anomalies under conditions of orthodontist shortage.

\section{MATERIALS AND METHODS}

We had carried out an analysis of research articles and academic issues, after which software to analyze control and diagnostic models of the jaws were created. To do this, we developed a software program where the points on the images were placed following the instructions. After that, it analyzes the models based on common methods. Later on we compared the calculation accuracy through matching the outcomes obtained by an analog method against those obtained by using our software program. After that the analysis was performed.

\section{RESULTS AND DISCUSSION}

One of the tasks within this work implied developing software to detect early signs of dental anomalies by analyzing control and diagnostic cast models in remote areas where residents cannot visit an orthodontist for various reasons.

The major features for the developed software were to be the following ones:

1) low system requirements;

2) user-friendly interface and easy using (the software is to be used by doctors of any profile);

3) minimum time spent by the doctor when using the software;

4) open source;

5) free distribution model.

The software allows performing analysis by using two photos of jaw cast models with minimal preliminary preparation and no serious technical support. It takes placing the required dots on the image, which is to be done following the detailed description appearing on the screen, after which the software will make its own calculations.

The software employs the main methods of analysis of control \& diagnostic models, which require data on the teeth size, on the distance between them and the length of the dental rows. The software requires 
measuring 1 centimeter on a ruler, and then, in the image, marking the most prominent points on the teeth mesial and distal surfaces of each jaw (Fig. 1).

After that, points are marked to identify the dentition width (Fig. 2). Further on, points are placed to identify the length of the apical basis, and then the software will require opening the models image in a frontal projection and place points to identify the apical basis width (Fig. 3).

When all the data is specified, the software displays the calculation outcomes based on the methods proposed by Pont, Linder-Harth, Gerlach, Bolton, Snagina, Korkhaus. The data is displayed in terms that are accessible to non-dental specialists (Fig. 4). Further options imply sending the report and save it on the PC.

The major differences between our development and those available on the market include:

1) free distribution;

2) easy to use; each stage of diagnostics offers graphic instructions in Russian;

3) low system requirements; requires 6 megabytes of free hard disk space;

4) can be used by doctors of any specialty.

The second stage in the development was to check the analysis accuracy compared to the conventional traditional method of jaw models anthropometry with a caliper.

The test involved ten undergraduate students majoring in Dentistry, who performed measurements on models - first in the traditional way, and then using the respective software. The results showed that the differences between the measurements fell within the range of $2-4 \%$, which is an acceptable tolerance.

The next step implied comparing the time spent on analog analysis and that using the software, which was done by untrained participants. We compared the time the students spent on analyzing 5 models in the traditional way and then - using our software. On average, the conventionally performed analysis took 35-42 minutes, whereas it took 3-4 minutes only to do that with the software, which, actually, means 8-10 times less than usual.

Putting the developed software to practice

1) Using this, as well as other similar developments, allows early detection of dental issues, which offers an obvious advantage since the successful outcome of treatment depends largely on the starting time of orthodontic treatment.

2) Many parents do not even suspect their children may be suffering from dental anomalies. Given that, another problem can be solved - the patient's awareness.

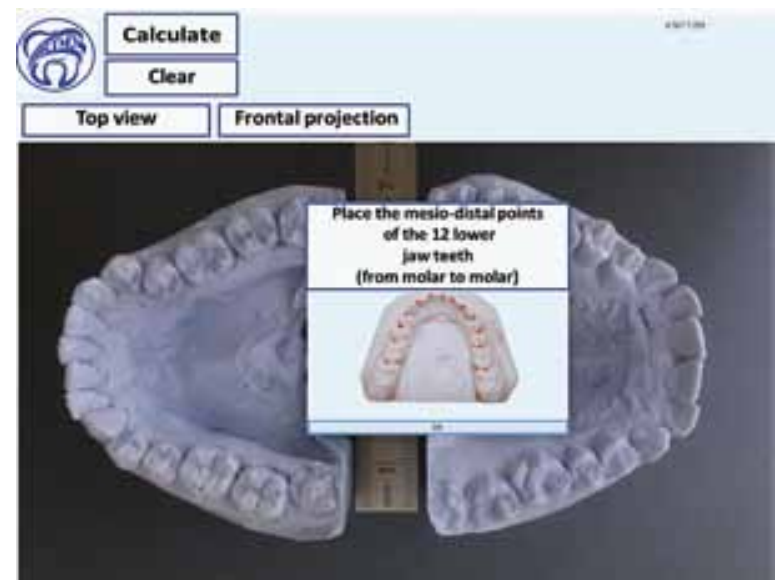

Fig.1. Example of a popup window with a step-by-step instruction

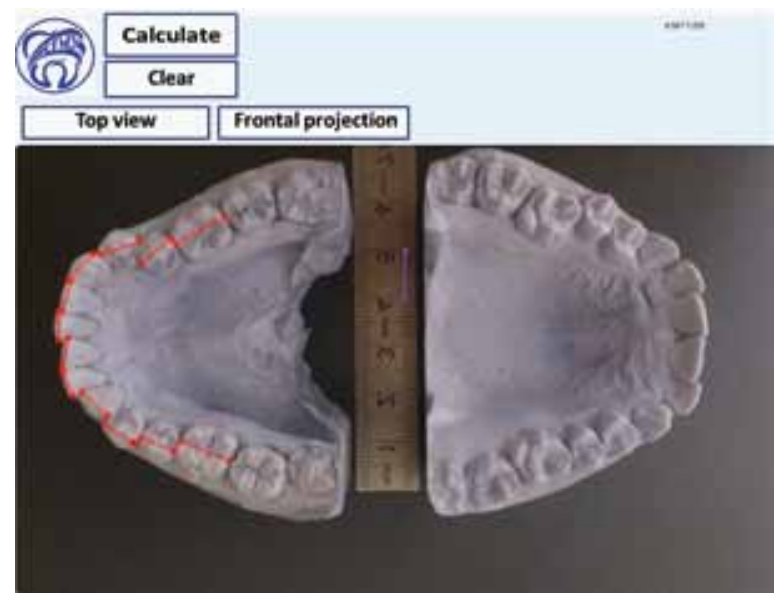

Fig. 2. Diagnostic models with points placed on the mesial and distal teeth surfaces, connected with reference lines

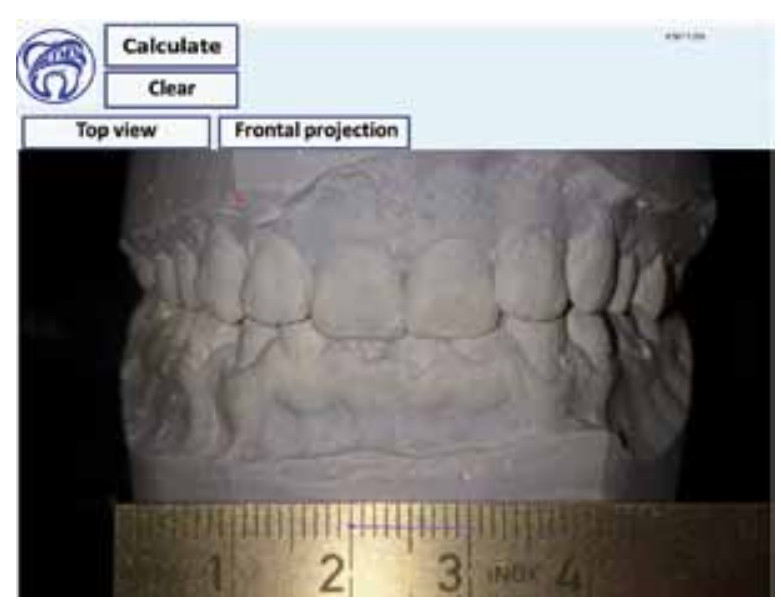

Fig. 3. Opening an image in a frontal projection to measure the apical basis 


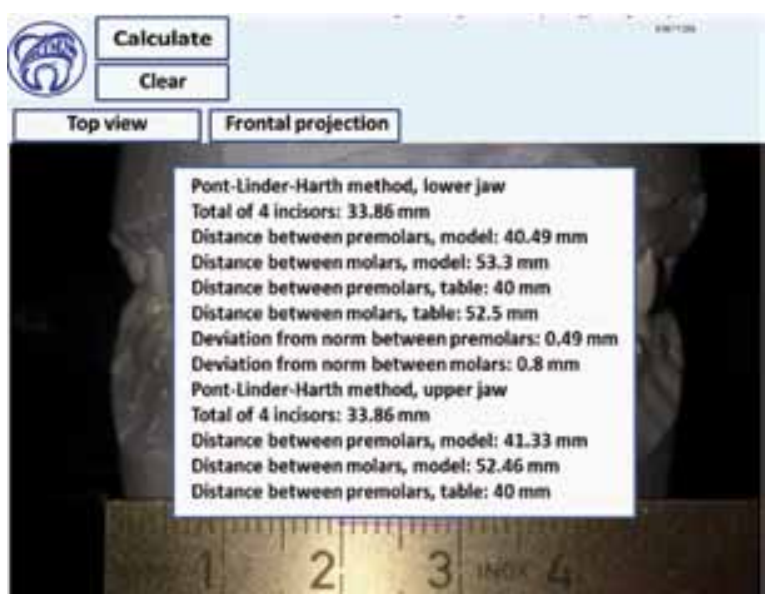

Fig. 4. Software compares measurements with table indices to make further conclusions

Table 1. Comparison of the measuring results for the four upper incisors sum by each student using the software and the conventional analysis

\begin{tabular}{|c|c|c|c|c|c|c|c|c|c|c|}
\hline Student & 1 & 2 & 3 & 4 & 5 & 6 & 7 & 8 & 9 & 10 \\
\hline 突总 & 28.5 & 28 & 28 & 29.5 & 28 & 28 & 28.5 & 28.5 & 28 & 29 \\
\hline 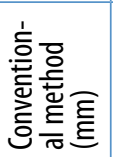 & 28 & 28.5 & 28 & 28.5 & 28.5 & 27 & 28 & 29.5 & 27 & 28.5 \\
\hline
\end{tabular}

3) Easy to use. Analyzing models using our software takes having 2 photos of models with a ruler for zooming.

4) Maximum simplification of the diagnostics procedure and minimization of the time spent on it.

5) Lower cost. Telemedicine technologies in orthodontics may allow saving not only the doctor's time, yet also the patients' money.

\section{O N CLUSION}

The proposed development allows analyzing models by a dentist who has no special skills. In view of a shortage of orthodontists to be observed in remote areas with a low population density, this software, if integrated into telemedicine systems, may facilitate detection of dental anomalies, at the same time possibly reducing the number of diagnostic errors.

\section{REFERENCES}

1. Firsova I.V., Suetenkov D.E., Egorova A.V., Magomedov T.B., KHARITONOVA T.L. Indicators of dental health in children and adolescents in Saratov and the Saratov region // Saratov Journal of Medical Scientific Research. 2013, T. 9; 3: 484-486

2. Estai, M., Kanagasingam, Y., Mehdizadeh, M.ET AL. Teledentistry as a novel pathway to improve dental health in school children: a research protocol for a randomised controlled trial. BMC Oral Health 20; 11 (2020). https://doi.org/10.1186/s12903-0190992-1

3. Suetenkov D.E., Kharitonova T.L., Danilov A.N., Popkova O.V., Kobets A.V. Dental morbidity in the school-age child population in the Saratov region. - Clinical dentistry. - 2019; 1: 96-99.

4. Nichols, K. (2019). Teledentistry Overview: United States of America. Journal of the International Society for Telemedicine and EHealth, 7, e9 (1-6). https:// doi.org/10.29086/JISfTeH.7.e9

5. Popkova O.V., Suetenkov D.E. Egorova A.V., Nasrullaev R.K. Telemedicine technologies for the clinic of dentistry (literature review) - Clinical dentistry. - 2018; 2: 93-96.

6. PARK, Jae. (2020). A licensed orthodontist versus doit-yourself orthodontics. American Journal of Orthodontics and Dentofacial Orthopedics. 157.591-592. DOI - 10.1016/j.ajodo.2020.02.003.

7. HarutyunYan Yu. Undifferentiated connective tissue dysplasia as a key factor in pathogenesis of maxillofacial disorders in children and adolescents // Archiv EuroMedica. 2020. Vol. 10; 2: 83-94. https://dx.doi. org/10.35630/2199-885X/2020/10/2.24

8. Kondratyeva T. Methodological approaches to dental arch morphology studying // Archiv EuroMedica. 2020. Vol. 10; 2: 95-100. https://dx.doi. org/10.35630/2199-885X/2020/10/2.25

9. ShKarin V.V., IVANov S.Yu., Lepilin A.V. Morphological specifics of craniofacial complex in people with varioustypes of facial skeleton growth in case of transversal occlusion anomalie // Archiv EuroMedica. 2019. Vol. 9; 2: 5-16. https://doi.org/10.35630/2199$885 \mathrm{X} / 2019 / 9 / 2 / 5$

10. Porfyriadis M.P. Scanning electron microscopy and X-ray spectral microanalysis in dental tissue resistance // Archiv EuroMedica. 2019. Vol. 9; 1: 177-185. https://doi.org/10.35630/2199-885X/2019/9/1/177

11. Shkarin V.V., Grinin V.M., Khalfin R.A. Specific features of transversal and vertical parameters in lower molars crowns at various dental types of arches // Archiv EuroMedica. 2019. Vol. 9; 2: 174-181. https:// doi.org/10.35630/2199-885X/2019/9/2/174 